Article

\title{
Fabrication of Pyramid Structure Substrate Utilized for Epitaxial Growth Free-Standing GaN
}

\author{
Ruixian Yu, Baoguo Zhang, Lei Zhang, Yongzhong Wu, Haixiao Hu, Lei Liu, Yongliang Shao * and \\ Xiaopeng Hao
}

State Key Lab of Crystal Materials, Shandong University, Jinan 250100, China; yuruixian0001@126.com (R.Y.); aoguo0909@163.com (B.Z.); leizhang528@sdu.edu.cn (L.Z.); wuyz@sdu.edu.cn (Y.W.);

huhaixiao0916@163.com (H.H.); liulei6560ailv@163.com (L.L.); xphao@sdu.edu.cn (X.H.)

* Correspondence: ylshao@sdu.edu.cn

Received: 9 September 2019; Accepted: 21 October 2019; Published: 23 October 2019

\begin{abstract}
Metal-organic chemical vapor deposition (MOCVD)-grown GaN on sapphire substrate was etched by hot phosphoric acids. Pyramid structures were obtained in the N-polar face of the MOCVD-GaN. Details of the formation process and morphology of the structures were discussed. The crystallographic plane index of the pyramid facet was calculated dependent on the symmetry of the wurtzite crystal structure and the tilt angle. The substrates with pyramid structures were utilized in subsequent hydride vapor phase epitaxy (HVPE) growth of GaN. Free-standing crystals were obtained, while HVPE-grown GaN achieved a certain thickness. Raman spectroscopy was employed to obtain the stress conditions of the HVPE-GaN without and with sapphire substrate. The mechanism of the self-separation process was discussed. This facile wet etching method may provide a simple way to acquire free-standing GaN by HVPE growth.
\end{abstract}

Keywords: etching; hydride vapor phase epitaxy; self-standing; gallium nitride; semiconductors

\section{Introduction}

In the last decade, many studies have been carried out in growing GaN for its advanced performance as a semiconductor material of optical and electrical devices, such as light-emitting diodes (LEDs) [1,2], laser diodes (LD) [3,4], UV detectors [5], and high-power, high-frequency electronic devices [6]. Due to the lack of native $\mathrm{GaN}$ substrates, $\mathrm{GaN}$ is usually grown on foreign substrates, such as sapphire, $\mathrm{SiC}, \mathrm{Si}$, etc. Lattice mismatch and thermal mismatch between $\mathrm{GaN}$ and the substrate result in large residual stress and high dislocation density [7,8]. Dislocations can form nonradiative recombination centers and light scattering centers, greatly reducing the luminous efficiency and lifetime of the GaN-based device. [9,10] To reduce the dislocation density, the in situ etched metal-organic chemical vapor deposition (MOCVD)-grown GaN template [11] or etched in molten salts template [12] were used to grow GaN crystals with the hydride vapor phase epitaxy (HVPE) method. The most effective way to reduce stress and dislocation is homoepitaxy on free-standing GaN substrate. Several methods were used to grow this kind of free-standing substrate. Removing the substrate of heteroepitaxy-grown GaN layer with laser lift-off technology is the most common method [13]. A TiN nanonet structure was used to fabricate a free-standing GaN layer by HVPE with a void-assisted separation process [14]. Williams and co-workers obtained free-standing GaN substrates after cooling through a natural separation process mechanism caused by the difference of thermal expansion coefficients between GaN and the sapphire substrate [15]. HVPE lateral overgrowth of thick GaN layers over masks of tungsten silicide nitride (WSiN) reduced sticking between the overgrown GaN and promoted self-separation of the overgrown layers [16]. Arrays of GaN nanorods with side-walls coated with silicon dioxide $\left(\mathrm{SiO}_{2}\right)$ were arranged randomly on the sapphire substrate as a growth 
template for subsequent HVPE and 2 in free-standing GaN substrates were fabricated [17]. However, these self-separating methods always introduce impurities into the growth system. At the same time, the processes before HVPE growing GaN are complex. In addition, the homo-epitaxy substrate also has residual strain and lattice distortion caused by HVPE heteroepitaxial growth; therefore, the surface nucleation and coalescence layer growth need to be optimized during the regrowth process. $[18,19]$ Lateral overgrowth can significantly reduce stress and dislocation density, which is a useful growth technique for growing high-quality GaN crystals. The facet structure is formed at the initial stage of growth. The lateral extension of the dislocations caused by facets contributes to the reduction of dislocation density. [20]

Some researchers reported the results of etching the N-polar face of GaN via different methods. Hock M. Ng et al. demonstrated the formation of GaN nanotip pyramids by selective and anisotropic etching of $\mathrm{N}$-polar $\mathrm{GaN}$ in $\mathrm{KOH}$ solution [21]. The plasma-assisted molecular beam epitaxy (MBE)-grown $\mathrm{GaN}$ was etched, and there were some hexagonal pyramids with six facets formed in the N-polar face. The lift-off freestanding GaN was etched by hot phosphoric acids, and dodecagonal pyramids could be obtained [22,23]. However, pyramid structures formed in the N-polar face of the MOCVD-GaN have not been investigated.

In this paper, we report a facile method to acquire free-standing GaN by HVPE on the etched MOCVD-GaN/sapphire substrate. The N-polar face of MOCVD-GaN was etched to form a unique pyramid morphology, which played an important role in the self-separating process of HVPE-grown GaN.

\section{Materials and Methods}

The substrates used in the HVPE process were 3-5 $\mu \mathrm{m}$ GaN layers fabricated by MOCVD on 2 in c-plane sapphire substrates. These substrates were etched in $\mathrm{H}_{3} \mathrm{PO}_{4}(85 \%)$ at $230{ }^{\circ} \mathrm{C}$ with different etching times. The subsequent epitaxy process was performed on the etched substrate after the cleaning process in a vertical HVPE growth system.

In order to investigate the etch effects, a field emission scanning electron microscope (FE-SEM, S-4800, Hitachi, Tokyo) was used to characterize the surface and cross-section morphology of the etched substrate. Raman spectroscopy was employed to obtain the stress conditions of the GaN crystal with and without substrate.

\section{Results}

Hexagonal pits appeared after the dislocation positions of GaN film were etched by $\mathrm{H}_{3} \mathrm{PO}_{4}$. Under different etching conditions, the obtained etch pits showed different morphology. The surface morphology of the samples with different etching times is shown in Figure 1. After 10 minutes of etching, an inverted hexagonal pyramid with six triangular facets forms (Figure 1a). The size and depth of the etch pits increase while extending the etching time. The etch pits penetrate the MOCVD-GaN layer and reach the sapphire substrates while the etching time is long enough. If the etching time continues to be extended, the etch pits expand to connect with each other. In this case, large irregularly shaped etch pits formed (Figure 1b). However, there were some inverted hexagonal pyramid still left under this very long etching time condition. 

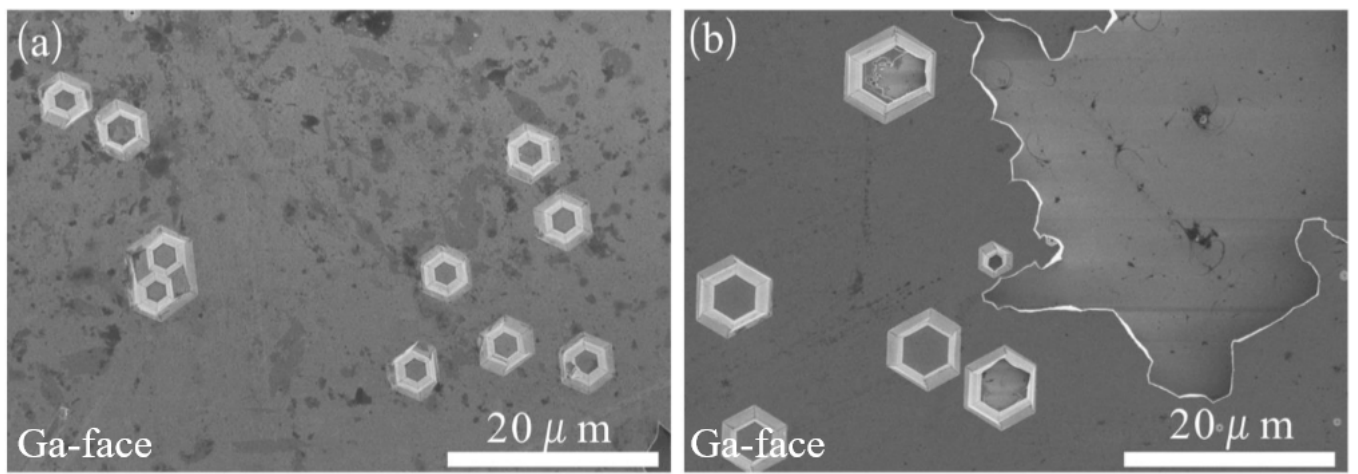

Figure 1. SEM images of the etched metal-organic chemical vapor deposition (MOCVD) grown GaN surface in $\mathrm{H}_{3} \mathrm{PO}_{4}$ at $230{ }^{\circ} \mathrm{C}$ for (a) $10 \mathrm{~min}$ and (b) $14 \mathrm{~min}$.

In order to confirm the details of the etch pit structures, the cross-section morphology of the etched substrates was characterized by SEM. In cross-section images, the MOCVD-GaN layer was etched to form some pyramid structures clustering together at the nitrogen-polar (N-polar) face (Figure 2a). These structures exist around the large irregularly shaped etch pits which reach the sapphire substrate (Figure 2b). This kind of pyramid structures will not be formed when the etch pits do not reach the substrate (Figure 2c). A bird's eye view image of the etched substrate also confirms these two kinds of etch pits (Figure 2d) in the MOCVD-GaN layer.
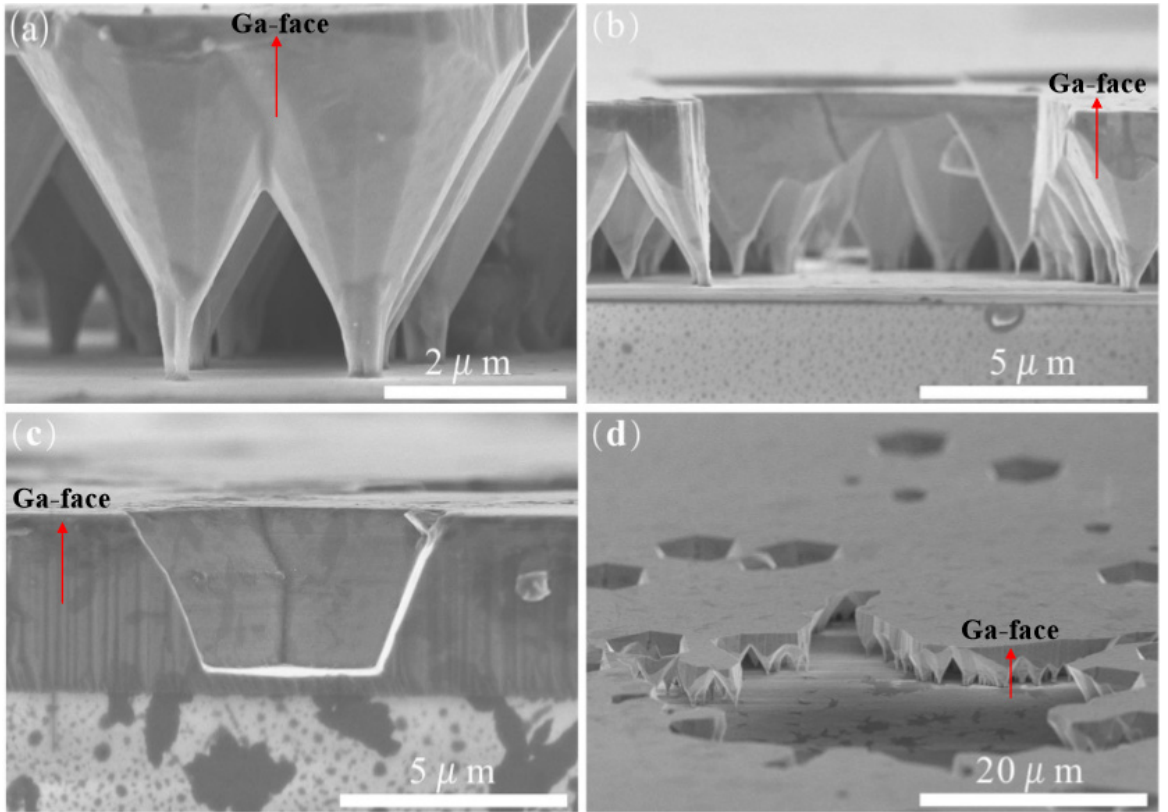

Figure 2. Cross-section SEM images of the MOCVD-grown GaN layer etched by phosphate for 14 min at $230{ }^{\circ} \mathrm{C}$. (a) The pyramid structures at the nitrogen polar (N-polar) face of MOCVD-GaN, (b) structures of $\mathrm{GaN}$ around the etch pit reach sapphire substrate, (c) the etch pit which does not reach sapphires substrate, and (d) a bird's eye view image of the etched substrate.

The pyramid structures were formed by etching the N-polar face of the MOCVD-GaN layer with hot phosphoric acids. In the case of long time etching to make the etch pit reach the sapphire substrate, the hot phosphoric acids etched $\mathrm{GaN}$ at the bottom of the etch pits to form these pyramid structures. However, for the etch pit which did not reach the sapphire substrate, extending the etching time only played a role in expanding the etch pit. The hot phosphoric acids did not reach and etch the N-polar face of MOCVD-GaN to form the pyramid structures. Extending the etching time so that many more etch pits reached the sapphire meant that the quantity of pyramid structures increased. 
The cross-section morphology of the pyramids (Figure 2a) shows that each of them is composed of several triangular facets, and on the tip, there is a very small column connected to the sapphire substrate. According to calculations, the angle between the inclined edge and the base of the pyramid is always $60-64^{\circ}$.

Dodecagonal pyramids are formed in the N-polar face of MOCVD-GaN after etching, and the twelve triangular facets belonging to two different sets of hexagonal facets with similar surface energies for the etching process are kinetic-limited (Figure 3a). The geometrical model of the pyramid consists of hexagonal facets and their mirror replicas [18]. In order to identify the facets of the dodecagonal pyramid, the intercept of the facet on three axes was calculated. The projection of the pyramid on (0001) is shown in Figure 3b. The red and green dotted lines represent the edges of two projections on (0001). The relationship between the intercept on a and $b$ direction is obtained in two triangles composed of $a, b$, and the edges of projections (Figure $3 b$ ).

In $\triangle \mathrm{OAC} \angle \mathrm{COA}=60^{\circ}, \angle \mathrm{OCA}=15^{\circ}, \angle \mathrm{OAC}=105^{\circ}$ :

$$
\mathrm{OC}=\mathrm{OA} \cdot \sin 30^{\circ}+\frac{\mathrm{OA} \cdot \sin 60^{\circ}}{\tan 15^{\circ}}=(\sqrt{3}+2) \cdot \mathrm{OA}=3.73 \cdot \mathrm{OA}
$$

In $\triangle \mathrm{OAB} \angle \mathrm{AOB}=120^{\circ}, \angle \mathrm{OAB}=45^{\circ}, \angle \mathrm{OBA}=15^{\circ}$ :

$$
\mathrm{OB}=\frac{\mathrm{OA} \cdot \sin 45^{\circ}}{\sin 15^{\circ}}=2.73 \cdot \mathrm{OA}
$$

The relationship between the intercept on a and c direction was obtained from the facet tilt angle $\alpha$.

$$
\begin{gathered}
\mathrm{OO}_{1}=\mathrm{OA}_{2} \cdot \cot \alpha \\
\mathrm{OO}_{1}=\mathrm{OA}_{2} \cdot \cot \alpha=\frac{2 \mathrm{OA}_{1}}{1+\sqrt{3}} \cdot \cot \alpha
\end{gathered}
$$

The intercept of these facets were calculated as follows:

$$
\begin{gathered}
1,-(2+\sqrt{3}), \cot \alpha \\
1, \sqrt{3}+1, \frac{1+\sqrt{3}}{2} \cdot \tan \alpha
\end{gathered}
$$

The index of the facet was described as the reciprocal of the intercept as:

$$
\begin{gathered}
\sqrt{3}+2,-1,(\sqrt{3}+2) \cdot \tan \alpha \\
\sqrt{3}+1,1,2 \tan \alpha
\end{gathered}
$$

The approximations of the plane indexes were $(4,-1,-3,-4)$ and $(3,1,-4,-4)$. The tilt angle of the facets was $59.4^{\circ}$. The stability of the GaN crystal plane is related to its surface energy, which is a function of the dangling bands density (DBD). The dangling bands density follow the order: Ga-face $<$ nonpolar face $<$ inclined face $<\mathrm{N}$ face [24]. Cabrera's thermodynamic theory shows that the strain energy of dislocations reduces the free energy of forming pits [25]. Therefore, etching occurs first at the position of the dislocation and then decomposes in the vertical direction ( $\mathrm{N}$ plane) of the dislocation while etching along the inclined surface. Therefore, the planes of $(4,-1,-3,-4),(3,1,-4$, $-4),(10-1-1)$ or (11-2-2), etc. are easily formed. Hence, the (10-1-1) and (11-2-2) planes may also be the exposed facets. 
(a) Top view

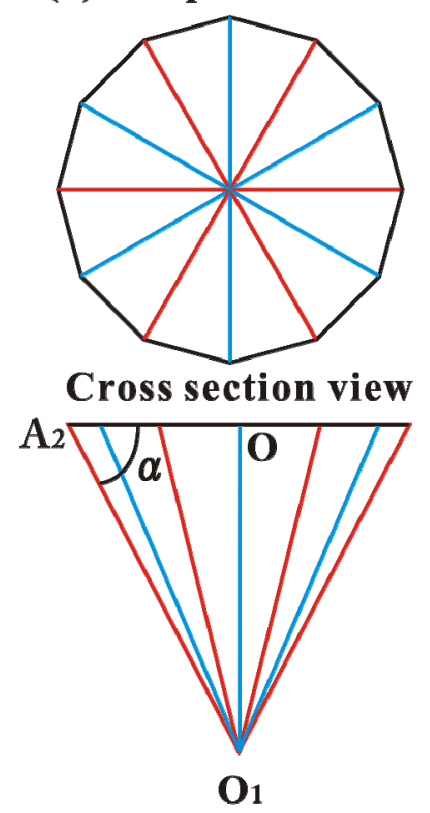

(b)

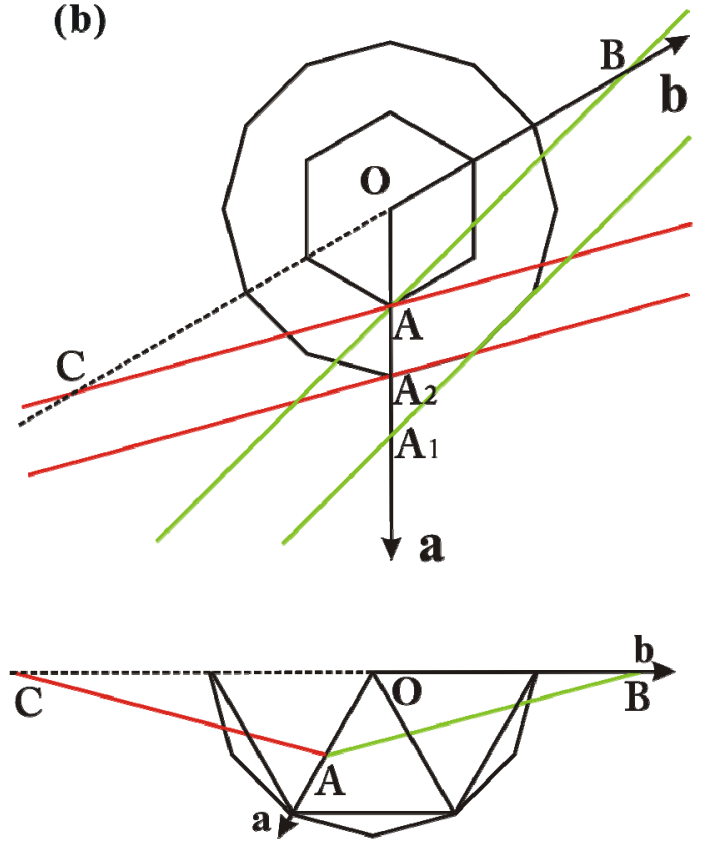

Figure 3. The schematic diagram of the dodecagonal pyramid with two different sets of (a) hexagonal facets and (b) the projection of the pyramid on (0001).

The MOCVD-grown GaN on sapphire was cleaned after the etching process, and then a vertical HVPE growth system was used to grow $\mathrm{GaN}$ on the etched substrate. The temperature of growing $\mathrm{GaN}$ was $1030^{\circ} \mathrm{C}$; the total reactor pressure during growth was atmosphere pressure. $\mathrm{GaCl}$ was formed from metallic $\mathrm{Ga}$ and $\mathrm{HCl}$ at $820^{\circ} \mathrm{C}$ with a flow rate of $50 \mathrm{sccm}$ and $\mathrm{NH}_{3}$ at a V/III of 20 for growth GaN. $\mathrm{N}_{2}$ was used as carrier gas. After cooling down to room temperature, a GaN layer with a thickness of more than $100 \mu \mathrm{m}$ was obtained. Strong residual stress caused by lattice mismatch and thermal expansion coefficient mismatch between GaN and sapphire substrate made the thick GaN layer crack. In this case, parts of GaN self-separate from the sapphire substrate (Figure 4a). In the region where the etch pits were not penetrated, the dislocations originating from the MOCVD-GaN substrate continued to extend upward in the HVPE-grown GaN crystal. To penetrate etching pits, the lateral epitaxial overgrowth extends over the etched pits, and the facet structures and voids are formed. The facets cause lateral extension of dislocations and reduce dislocation density. The void releases the stress of the grown GaN crystal. Some free-standing GaN crystals are obtained (Figure 4b). The morphology of the free-standing HVPE-GaN N-polar face was characterized by SEM. At the location of each large irregularly shaped etch pit, there is a terrace in the canter (Figure 4c). The terrace was formed by the growth of GaN to fill the large irregularly shaped etch pit. Contrasted with the morphology of the etched N-polar face of MOCVD-GaN before HVPE growth, the dodecagonal pyramid disappears, but some pyramids with different shapes around the terrace are observed (Figure 4d). The shape of the pyramids changes from twelve facets to six facets. It is speculated that the N-polar face growth of the two sets of facets is caused by the supply of source gas in the HVPE growth process. 


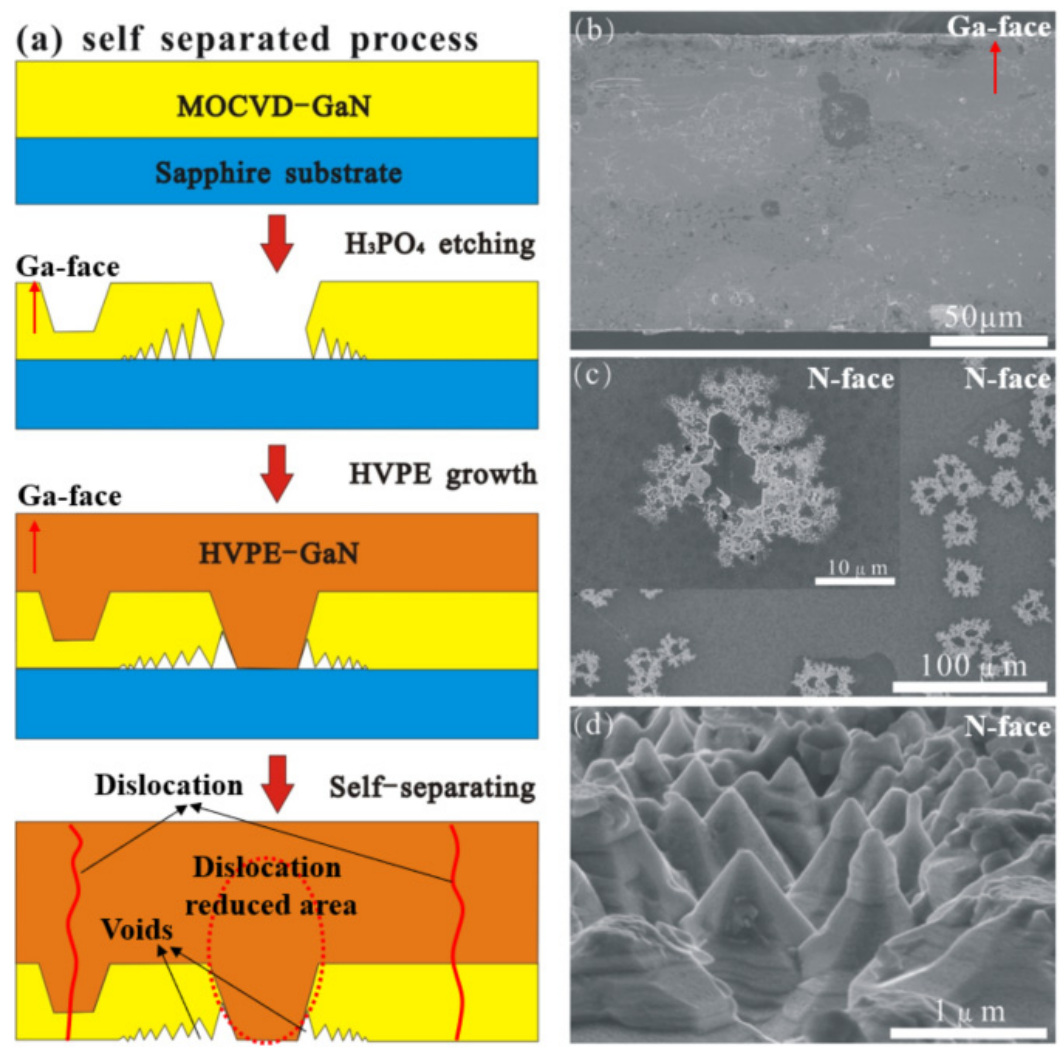

Figure 4. The schematic diagram of (a) the self-separating process. (b) Cross-section SEM image of the free-standing GaN crystal. (c) SEM image of the free-standing hydride vapor phase epitaxy (HVPE)-GaN N-polar face. (d) After HVPE growth, the pyramids show six facets.

The pyramid structures in the N-polar face of MOCVD-GaN reduced the contact area between epitaxial GaN and sapphire substrate and formed many voids in the N-polar face of HVPE-GaN. Raman spectroscopy was carried out to obtain the stress condition. The $\mathrm{E}_{2}$ (high) and $\mathrm{A}_{1}$ (LO) modes were detected in free-standing $\mathrm{GaN}$ and $\mathrm{GaN}$ with sapphire substrate. It has been reported that the relationship between stress and the shift of $\mathrm{E}_{2}$ (high) Raman mode can be obtained using the following equation [19]:

$$
\sigma=\Delta \omega / 4.2\left(\mathrm{~cm}^{-1} \mathrm{GPa}^{-1}\right)
$$

where $\sigma$ is the stress (GPa) and $\Delta \omega$ is the $\mathrm{E}_{2}$ (high) mode peak shift $\left(\mathrm{cm}^{-1}\right)$. The wave number of $\mathrm{E}_{2}$ (high) mode of the HVPE-GaN without and with sapphire substrate is detected at $566.5 \mathrm{~cm}^{-1}$ and $568.2 \mathrm{~cm}^{-1}$, respectively (Figure 5). Considering that the E2 (high) Raman mode of stress-free standard GaN [26] has a wave number of $566.2 \mathrm{~cm}^{-1}$, the residual compressive stress of GaN (0.36 GPa) grown on etched substrate is smaller than that of the GaN grown on unetched (0.48 GPa) substrate. This proves that this kind of pyramid structure is beneficial to reduce stress. 


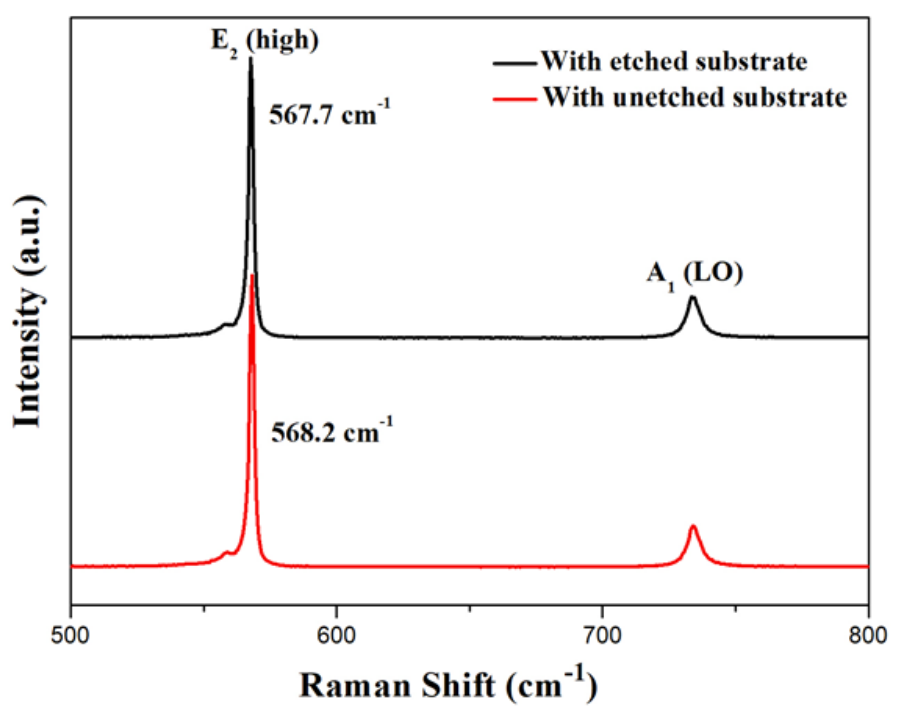

Figure 5. Raman spectra of HVPE-GaN with and without substrate. The wave number of $\mathrm{E}_{2}$ (high) mode of the HVPE-GaN grown on etched and unetched substrate is detected at $567.7 \mathrm{~cm}^{-1}$ and $568.2 \mathrm{~cm}^{-1}$, respectively.

\section{Conclusions}

In summary, hexagonal etch pits were formed in the MOCVD-grown GaN on sapphire after being etched in hot phosphoric acids. With a long etching time, the etch pits reached the sapphire substrate, and when we kept extending the etching time, the etch pits connected with each other to form large irregularly shaped etch pits. There were some pyramid structures in the N-polar face of MOCVD-GaN clustered around the etch pits reaching the sapphire substrate. These pyramids had twelve facets. The crystallographic plane indexes of the facets were identified as $(4,-1,-3,-4)$ and $(3$, $1,-4,-4)$ according to the symmetry of the wurtzite structure GaN. This kind of structure reduced the contact area between epitaxial $\mathrm{GaN}$ and sapphire substrate and was beneficial to the self-separating process. This wet etching method provides a possible facile method to obtain free-standing $\mathrm{GaN}$ using HVPE growth.

Author Contributions: X.P. and Y.Z. designed the experiment. Y.L. and R.X. wrote the main manuscript text. L.Z., B.G., H.X., and L.L. helped with the sample fabrication. All the authors participated in the discussion and revision of the manuscript.

Funding: This research was funded by the National Natural Science Foundation of China (Contract No. 51602177, 51872162, 51872164).

Acknowledgments: This work was supported by the National Natural Science Foundation of China (Contract No. 51602177, 51872162, 51872164).

Conflicts of Interest: The authors declare no conflict of interest.

\section{References}

1. Nakamura, S.; Mukai, T.; Senoh, M. Candela-class high-brightness InGaN/AlGaN double-heterostructure blue-light-emitting diodes. Appl. Phys. Lett. 1994, 64, 1687-1689. [CrossRef]

2. Nakamura, S.; Senoh, M.; Iwasa, N.; Nagahama, S. High-Brightness InGaN Blue, Green and Yellow Light-Emitting Diodes with Quantum Well Structures. Jpn. J. Appl. Phys. 1995, 34, L797-L799. [CrossRef]

3. Akasaki, I.; Sota, S.; Sakai, H.; Tanaka, T.; Koike, M.; Amano, H. Shortest wavelength semiconductor laser diode. Electron. Lett. 1996, 32, 1105-1106. [CrossRef] 
4. Nakamura, S.; Senoh, M.; Nagahama, S.I.; Iwasa, N.; Yamada, T.; Matsushita, T.; Kiyoku, H.; Sugimoto, Y.; Kozaki, T.; Umemoto, H.; et al. InGaN/GaN/AlGaN-based laser diodes with modulation-doped strained-layer superlattices. Jpn. J. Appl. Phys. 1997, 36, L1568-L1571. [CrossRef]

5. Asif Khan, M.; Kuznia, J.N.; Olson, D.T.; Schaff, W.J.; Burm, J.W.; Shur, M.S. Microwave performance of a 0.25 $\mu \mathrm{m}$ gate $\mathrm{AlGaN} / \mathrm{GaN}$ heterostructure field effect transistor. Appl. Phys. Lett. 1994, 65, 1121-1123. [CrossRef]

6. Sandvik, P.; Mi, K.; Shahedipour, F.; McClintock, R.; Yasan, A.; Kung, P.; Razeghi, M. $\mathrm{Al}_{\mathrm{x}} \mathrm{Ga}_{1-\mathrm{x}} \mathrm{N}_{\text {for }}$ solar-blind UV detectors. J. Cryst. Growth 2001, 231, 366-370. [CrossRef]

7. Kapolnek, D.; Wu, X.H.; Heying, B.; Keller, S.; Keller, B.P.; Mishra, U.K.; DenBaars, S.P.; Speck, J.S. Structural evolution in epitaxial metalorganic chemical vapor deposition grown GaN films on sapphire. Appl. Phys. Lett. 1995, 67, 1541-1543. [CrossRef]

8. Qian, W.; Skowronski, M.; De Graef, M.; Doverspike, K.; Rowland, L.B.; Gaskill, D.K. Microstructural characterization of $\alpha-\mathrm{GaN}$ films grown on sapphire by organometallic vapor phase epitaxy. Appl. Phys. Lett. 1995, 66, 1252-1254. [CrossRef]

9. Amano, H. Progress and prospect of the growth of wide-band-gap group III nitrides: development of the growth method for single-crystal bulk GaN. Jpn. J. Appl. Phys. 2013, 52, 050001. [CrossRef]

10. Zhang, L; Li, X.; Shao, Y.L.; Yu, J.X.; Wu, Y.Z.; Hao, X.P.; Yin, Z.M.; Dai, Y.B.; Tian, Y.; Huo, Q.; et al. Improving the quality of $\mathrm{GaN}$ crystals by using graphene or hexagonal boron nitride nanosheets substrate. Appl. Mater. Interfaces 2015, 7, 4504-4510. [CrossRef]

11. Lin, C.; Yu, G.; Wang, X.; Cao, M.; Lu, H.; Gong, H.; Qi, M.; Li, A. Hydride vapor phase epitaxy growth of high-quality GaN film on in situ etched GaN template. Mater. Lett. 2009, 63, 943-945. [CrossRef]

12. Weyher, J.L.; Ashraf, H.; Hageman, P.R. Reduction of dislocation density in epitaxial GaN layers by overgrowth of defect-related etch pits. Appl. Phys. Lett. 2009, 95, 031913. [CrossRef]

13. Kelly, M.K.; Ambacher, O.; Dimitrov, R.; Handschuh, R.; Stutzmann, M. Optical Process for Liftoff of Group III-Nitride Films. Phys. Status Solidi A 1997, 159, R3-R4. [CrossRef]

14. Oshima, Y.; Shibata, T.; Eri, M.; Sunakawa, H.; Usui, A. Fabrication of freestanding GaN wafers by Hydride Vapor-Phase Epitaxy with void-assisted separation. Phys. Status Solidi A 2002, 194, 554-558. [CrossRef]

15. Williams, A.D.; Moustakas, T.D. Formation of large-area freestanding gallium nitride substrates by natural stress-induced separation of GaN and sapphire. J. Cryst. Growth 2007, 300, 37-41. [CrossRef]

16. Hennig, Ch.; Richter, E.; Weyers, M.; Tränkle, G. Freestanding 2-in GaN layers using lateral overgrowth with HVPE. J. Cryst. Growth 2008, 310, 911-915. [CrossRef]

17. Chao, C.L.; Chiu, C.H.; Lee, Y.J.; Kuo, H.C.; Liu, P.C.; Tsay, J.D.; Cheng, S.J. Freestanding high quality GaN substrate by associated GaN nanorods self-separated hydride vapor-phase epitaxy. Appl. Phys. Lett. 2009, 95, 051905. [CrossRef]

18. Liu, N.L.; Cheng, Y.T.; Wu, J.J.; Li, X.B.; Yu, T.B.; Xiong, H.; Li, W.H.; Chen, J.; Zhang, G.Y. HVPE homoepitaxial growth of high quality bulk GaN using acid wet etching method and its mechanism analysis. J. Cryst. Growth 2016, 454, 59-63. [CrossRef]

19. Bockowski, M.; Iwinska, M.; Amilusik, M.; Fijalkowski, M.; Lucznik, B.; Sochacki, T. Challenges and future perspectives in HVPE-GaN growth on ammonothermal GaN seeds. Semicond. Sci. Technol. 2016, 31, 093002. [CrossRef]

20. Hiramatsu, K. Epitaxial lateral overgrowth techniques used in group III nitride epitaxy. J. Phys. Condens. Matter 2001, 13, 6961-6975. [CrossRef]

21. Ng, H.M.; Weimann, N.G.; Chowdhury, A. GaN nanotip pyramids formed by anisotropic etching. J. Appl. Phys. 2003, 94, 650. [CrossRef]

22. Qi, S.L.; Chen, Z.Z.; Fang, H.; Sun, Y.J.; Sang, L.W.; Yang, X.L.; Zhao, L.B.; Tian, P.F.; Deng, J.J.; Tao, Y.B.; et al. Study on the formation of dodecagonal pyramid on nitrogen polar $\mathrm{GaN}$ surface etched by hot $\mathrm{H}_{3} \mathrm{PO}_{4}$. Appl. Phys. Lett. 2009, 95, 071114. [CrossRef]

23. Yu, F.; Chen, Z.; Qi, S.L.; Wang, S.; Jiang, S.; Fu, X.; Jiang, X.; Yu, T.; Qin, Z.; Kang, X.; et al. Changing oblique angles of pyramid facets fabricated by wet etching of N polar GaN. CrystEngComm 2012, 14, 4781-4785. [CrossRef]

24. Yu, J.; Zhang, L.; Shen, J.; Xiu, Z.; Liu, S. Wafer-scale porous GaN single crystal substrates and its application in energy storage. CrystEngComm 2016, 18, 18-5154. [CrossRef] 
25. Zhang, L.; Shao, Y.L.; Wu, Y.Z.; Hao, X.P.; Chen, X.F.; Qu, S.; Xu, X.G. Characterization of dislocation etch pits in HVPE-grown GaN using different wet chemical etching methods. J. Alloys Compd. 2010, 504, 186-191. [CrossRef]

26. Kisielowski, C.; Krüger, J.; Ruvimov, S.; Suski, T.; Ager, J.W.; Jones, E.; Liliental-Weber, Z.; Rubin, M.; Weber, E.R.; Bremser, M.D.; et al. Strain-related phenomena in GaN thin films. Phys. Rev. B 1996, 54, 17745. [CrossRef]

(c) ( (C) 2019 by the authors. Licensee MDPI, Basel, Switzerland. This article is an open access article distributed under the terms and conditions of the Creative Commons Attribution (CC BY) license (http://creativecommons.org/licenses/by/4.0/). 